International Conference Mathematical and Computational Biology 2011

International Journal of Modern Physics: Conference Series

Vol. 9 (2012) 529-536

C World Scientific Publishing Company

DOI: $10.1142 / \mathrm{S} 2010194512005624$

\title{
EVASION FROM ONE PURSUER IN A HILBERT SPACE
}

\author{
FATEH ALLAHABI \\ Department of Mathematics, Universiti Putra Malaysia, \\ UPM Serdang, Selangor 43400, Malaysia \\ fateh@math.upm.edu.my \\ IBRAGIMOV G.I. \\ Institute for Mathematical Research \& \\ Department of Mathematics, Universiti Putra Malaysia, \\ UPM Serdang, Selangor 43400, Malaysia \\ gafur@science.upm.edu.my
}

\begin{abstract}
We study a differential game of one pursuer and one evader described by infinite systems of second order ordinary differential equations. Controls of players are subjected to geometric constraints. Differential game is considered in Hilbert spaces. We proved one theorem on evasion. Moreover, we constructed explicitly a control of the evader.
\end{abstract}

Keywords: Differential game; pursuer; evader; control; evasion.

\section{Introduction}

Differential game problems for systems described by partial differential equations are of increasing interest (See Refs. 2, 3 and 5-8). Some optimal control and differential game problems for the partial differential equations can be reduced to the one described by infinite systems of ordinary differential equations by using the decomposition method (See Refs. 2, 3). The same method can be applied to solve differential game problems for such equations (See Refs. 5-9). Hence there is a significant relationship between control and differential game problems described by partial differential equations and those described by infinite system of differential equations. The latter seems to be of independent interest and can be investigated within one theoretical framework. In (Ref. 9), an evasion differential game described by infinite system of differential equations with finite number of pursuers was studied under geometric constraints on controls. In the present paper, we study an evasion differential game of one pursuer and one evader described by infinite system of 2-systems of second order. We present a theorem on evasion for such system in the Hilbert space $l_{r}^{2}$. Geometric constraints are imposed on control functions of players. The goal of the pursuer is to force the system and its velocity to the origin of the spaces $l_{r+1}^{2}$ and $l_{r}^{2}$ respectively, and the evader exactly tries to avoid this. 


\section{Statement of Problem}

Let $\lambda_{1}, \lambda_{2}, \ldots$ be a sequence of positive numbers, and $r$ be a fixed number. We introduce into the consideration the space

$$
l_{r}^{2}=\left\{\xi=\left(\xi_{1}, \xi_{2}, \ldots\right): \sum_{i=1}^{\infty} \lambda_{i}^{r}\left|\xi_{i}\right|^{2}<\infty\right\}, \xi_{i} \in R^{2},
$$

with the inner product and norm

$$
\langle\xi, \eta\rangle=\sum_{i=1}^{\infty} \lambda_{i}^{r} \xi_{i} \eta_{i}, \xi, \eta \in l_{r}^{2},\|\xi\|_{l_{r}^{2}}=\left(\sum_{i=1}^{\infty} \lambda_{i}^{r}\left|\xi_{i}\right|^{2}\right)^{1 / 2} .
$$

Denote

$$
D_{k}=\left[\begin{array}{rr}
-\alpha_{k} & -\beta_{k} \\
\beta_{k} & -\alpha_{k}
\end{array}\right], \quad \alpha_{k}, \beta_{k} \in \mathrm{R}, k=1,2, \ldots
$$

Consider the differential game described by countably many differential equations

$$
\ddot{z}_{k}=D_{k} z_{k}-u_{k}+v_{k}, z_{k}(0)=z_{k}^{0}, \dot{z}_{k}(0)=z_{k}^{1}, k=1,2, \ldots,
$$

where $z_{k}, u_{k}, v_{k}, z_{k}^{0}, z_{k}^{1} \in R^{2}, z^{0}=\left(z_{1}^{0}, z_{2}^{0}, \ldots\right) \in l_{r+1}^{2}, z^{1}=\left(z_{1}^{1}, z_{2}^{1}, \ldots\right) \in l_{r}^{2}$,

$$
\left\|z^{0}\right\|_{r+1}+\left\|z^{1}\right\|_{r} \neq 0
$$

$u=\left(u_{1}, u_{2}, \ldots\right)$ is control parameter of the pursuer, and $v=\left(v_{1}, v_{2}, \ldots\right)$ is that of the evader. In the sequel, we consider $\lambda_{k}=\sqrt{\alpha_{k}^{2}+\beta_{k}^{2}}, k=1,2, \ldots$

Let $L_{2}\left(0, T ; l_{r}^{2}\right)$ be the space of functions $f(t)=\left(f_{1}(t), f_{2}(t), \ldots\right), f:[0, T] \rightarrow l_{r}^{2}$, with measurable coordinates $f_{k}(t)=\left(f_{k 1}(t), f_{k 2}(t)\right), 0 \leq t \leq T$, such that

$$
\|f(\cdot)\|_{L_{2}\left(0, T ; l_{r}^{2}\right)}=\sum_{k=1}^{\infty} \lambda_{k}^{r} \int_{0}^{T}\left(f_{k 1}^{2}(t)+f_{k 2}^{2}(t)\right) d t<\infty,
$$

where $T$ is a given positive number.

Definition 1. A function $u(\cdot)=\left(u_{1}(\cdot), u_{2}(\cdot), \ldots\right) \in L_{2}\left(0, T ; l_{r}^{2}\right)$ satisfying the condition

$$
\sum_{k=1}^{\infty} \lambda_{k}^{r}\left|u_{k}\right|^{2} \leq \rho^{2}
$$

where $\rho$ is a given positive number, is called an admissible control of the pursuer.

Definition 2. A function $v(\cdot)=\left(v_{1}(\cdot), v_{2}(\cdot), \ldots\right) \in L_{2}\left(0, T ; l_{r}^{2}\right)$ satisfying the condition 


$$
\sum_{k=1}^{\infty} \lambda_{k}^{r}\left|v_{k}\right|^{2} \leq \sigma^{2}
$$

where $\sigma$ is a given positive number, is called an admissible control of the evader.

Let

$$
\begin{gathered}
A_{k 1}(t)=e^{r_{1 k} t}\left[\begin{array}{ll}
\cos \left(r_{2 k} t\right) & -\sin \left(r_{2 k} t\right) \\
\sin \left(r_{2 k} t\right) & \cos \left(r_{2 k} t\right)
\end{array}\right], A_{k 2}(t)=A_{k 1}(-t), R_{k}=\left[\begin{array}{ll}
r_{1 k} & -r_{2 k} \\
r_{2 k} & r_{1 k}
\end{array}\right], \\
A_{k}(t)=\frac{1}{2}\left(A_{k 1}(t)+A_{k 2}(t)\right), B_{k}(t)=\frac{1}{2} R_{k}^{-1}\left(A_{k 1}(t)-A_{k 2}(t)\right), \\
r_{1 k}=\sqrt{\frac{-\alpha_{k}+\sqrt{\alpha_{k}^{2}+\beta_{k}^{2}}}{2}}, r_{2 k}=\sqrt{\frac{\alpha_{k}+\sqrt{\alpha_{k}^{2}+\beta_{k}^{2}}}{2}}, k=1,2, \ldots
\end{gathered}
$$

Clearly, $r_{k}=\sqrt{r_{1 k}^{2}+r_{2 k}^{2}}=\sqrt[4]{\alpha_{k}^{2}+\beta_{k}^{2}}=\sqrt{\lambda_{k}}, k=1,2, \ldots$

It can be easily proved that

$$
\begin{aligned}
& A_{k}^{2}(t)-R_{k}^{2} B_{k}^{2}(t)=E_{2}, \\
& \quad A_{k}(t) B_{k}(t-s)-B_{k}(t) A_{k}(t-s)=-B_{k}(s), \\
& A_{k}(t) A_{k}(t-s)-R_{k}^{2} B_{k}(t) B_{k}(t-s)=A_{k}(s) .
\end{aligned}
$$

Let $C\left(0, T ; l_{r}^{2}\right)$ be the space of continuous functions $z(t), 0 \leq t \leq T$, with the values in the space $l_{r}^{2}$. If $\left\{r_{1 k}\right\}_{k \in N}$ is a bounded above sequence, then the infinite system of differential equations

$$
\ddot{z}_{k}(t)=D_{k} z_{k}(t)+w_{k}(t), z_{k}(0)=z_{k}^{0}, \dot{z}_{k}(0)=z_{k}^{1}, k=1,2, \ldots
$$

has a unique solution $z(\cdot) \in C\left(0, T ; l_{r+1}^{2}\right)$ defined by

$$
z_{k}(t)=A_{k}(t) z_{k 0}+B_{k}(t) z_{k 1}+\int_{0}^{t} B_{k}(t-s) w_{k}(s) d s, k=1,2, \ldots
$$

It can be verified that

$$
\left.\dot{z}_{k}(t)=R_{k}^{2} B_{k}(t) z_{k 0}+A_{k}(t) z_{k 1}+\int_{0}^{t} A_{k}(t-s) w_{k}(s)\right) d s .
$$

(Ref. 10). 
Definition 4. We say that evasion is possible in the game (1) from an initial position $\left\{z^{0}, z^{1}\right\}$, where $z^{0}=\left(z_{1}^{0}, z_{2}^{0}, \ldots\right) \in l_{r+1}^{2}, z^{1}=\left(z_{1}^{1}, z_{2}^{1}, \ldots\right) \in l_{r}^{2}$, if it can be chosen an admissible control $v(\cdot)$ of the evader such that for any admissible control of pursuer $u(\cdot)$, and arbitrary number $\vartheta>0$, the relation $\|z(t)\|_{r+1}+\|\dot{z}(t)\|_{r} \neq 0$ is true for all $t \in[0, \vartheta]$.

Problem. Find conditions for the evasion to be possible.

\section{Main Result}

In this sections we present a theorem on evasion.

Theorem 3.1. If $\rho \leq \sigma$ then from any initial position $\left\{z^{0}, z^{1}\right\}, z^{0}=\left(z_{1}^{0}, z_{2}^{0}, \ldots\right) \in l_{r+1}^{2}, z^{1}=$ $\left(z_{1}^{1}, z_{2}^{1}, \ldots\right) \in l_{r}^{2},\|z(t)\|_{r+1}+\|\dot{z}(t)\|_{r} \neq 0$, evasion is possible in the game (1).

Proof. Let $\vartheta$ be an arbitrary positive number. Consider the following infinite system

$$
\left\{\begin{array}{l}
z_{k}(t)=A_{k}(t) z_{k}^{0}+B_{k}(t) z_{k}^{1}+\int_{0}^{t} B_{k}(t-s) w_{k}(s) d s, \\
\left.\dot{z}_{k}(t)=R_{k}^{2} B_{k}(t) z_{k}^{0}+A_{k}(t) z_{k}^{1}+\int_{0}^{t} A_{k}(t-s) w_{k}(s)\right) d s,
\end{array}\right.
$$

where $w_{k}(t)=-u_{k}(t)+v_{k}(t), 0 \leq t \leq T, k=1,2, \ldots$.

In order to obtain a more simple system we transform (8) by setting

$$
\left[\begin{array}{l}
x_{k}(t) \\
y_{k}(t)
\end{array}\right]=\left[\begin{array}{ll}
A_{k}(t) & -R_{k} B_{k}(t) \\
-R_{k} B_{k}(t) & A_{k}(t)
\end{array}\right]\left[\begin{array}{l}
R_{k} z_{k}(t) \\
\dot{z}_{k}(t)
\end{array}\right],\left[\begin{array}{l}
x_{k}^{0} \\
y_{k}^{0}
\end{array}\right]=\left[\begin{array}{l}
R_{k} z_{k}^{0} \\
z_{k}
\end{array}\right], \quad k=1,2, \ldots .
$$

Consequently,

$$
\begin{aligned}
x_{k}(t)= & R_{k} A_{k}(t) z_{k}(t)-R_{k} B_{k}(t) \dot{z}_{k}(t) \\
= & R_{k}\left(A_{k}^{2}(t)-R_{k}^{2} B_{k}^{2}(t)\right) z_{k}^{0}+R_{k}\left(A_{k}(t) B_{k}(t)-B_{k}(t) A_{k}(t)\right) z_{k}^{1} \\
& \left.+\int_{0}^{t} R_{k}\left(A_{k}(t) B_{k}(t-s)-B_{k}(t) A_{k}(t-s)\right) w_{k}(s)\right) d s,
\end{aligned}
$$

and

$$
\begin{aligned}
y_{k}(t)= & -R_{k}^{2} B_{k}(t) z_{k}(t)+A_{k}(t) \dot{z}_{k}(t) \\
= & \left(-R_{k}^{2} B_{k}(t) A_{k}(t)+A_{k}(t) R_{k}^{2} B_{k}(t)\right) z_{k}^{0}+\left(-R_{k}^{2} B_{k}^{2}(t)+A_{k}^{2}(t)\right) z_{k}^{1} \\
& \left.+\int_{0}^{t}\left(-R_{k}^{2} B_{k}(t) B_{k}(t-s)+A_{k}(t) A_{k}(t-s)\right) w_{k}(s)\right) d s .
\end{aligned}
$$

By using (4), (5) and (6), we obtain 


$$
\left\{\begin{array}{l}
\left.x_{k}(t)=x_{k}^{0}-\int_{0}^{t} R_{k} B_{k}(s) w_{k}(s)\right) d s, \\
\left.y_{k}(t)=y_{k}^{0}+\int_{0}^{t} A_{k}(s) w_{k}(s)\right) d s,
\end{array} \quad k=1,2, \ldots,\right.
$$

where

$$
x_{k}^{0} \in R^{2}, y_{k}^{0} \in R^{2}, \quad x^{0}=\left(x_{1}^{0}, x_{2}^{0}, \ldots\right) \in l_{r}^{2}, y^{0}=\left(y_{1}^{0}, y_{2}^{0}, \ldots\right) \in l_{r}^{2} .
$$

Further, we consider the system (10). As,

$$
\left\|x^{0}\right\|_{r}+\left\|y^{0}\right\|_{r} \neq 0
$$

then we can pick a natural number $M$ such that the $2 M$-dimensional vectors

$$
X^{0}=\left(x_{1}^{0}, x_{2}^{0}, \ldots, x_{M}^{0}\right) \in R^{2 M}, Y^{0}=\left(y_{1}^{0}, y_{2}^{0}, \ldots, y_{M}^{0}\right) \in R^{2 M},
$$

satisfy the relations

$$
\left|X^{0}\right|+\left|Y^{0}\right| \neq 0
$$

that is vectors $X^{0}$ and $Y^{0}$ as elements of the space $R^{2 M}$ are not equal to zero simultaneously. Therefore there exist a unit vectors $p, q \in R^{2 M}, p=\left(p_{1}, p_{2}, \ldots, p_{M}\right)$, $p_{i}=\left(p_{i 1}, p_{i 2}\right), \quad q=\left(q_{1}, q_{2}, \ldots, q_{M}\right), q_{i}=\left(q_{i 1}, q_{i 2}\right), \quad i=1,2, \ldots, M$, such that

$$
\left\langle p, X^{0}\right\rangle \geq 0,\left\langle q, Y^{0}\right\rangle \geq 0
$$

Instead of (9) we consider the following finite systems of equations

$$
\left\{\begin{array}{l}
\left.\left.X_{k}(t)=x_{k}^{0}+\int_{0}^{t} R_{k} B_{k}(s) u_{k}(s)\right) d s-\int_{0}^{t} R_{k} B_{k}(s) v_{k}(s)\right) d s \\
\left.\left.Y_{k}(t)=y_{k}^{0}-\int_{0}^{t} A_{k}(s) u_{k}(s)\right) d s+\int_{0}^{t} A_{k}(s) v_{k}(s)\right) d s,
\end{array} \quad k=1,2, \ldots, M\right.
$$

where $X^{0}=\left(x_{1}^{0}, x_{2}^{0}, \ldots x_{M}^{0}\right), Y^{0}=\left(y_{1}^{0}, y_{2}^{0}, \ldots y_{M}^{0}\right)$, with control parameters satisfying the conditions

$$
\sum_{k=1}^{M} \lambda_{k}^{r} u_{k}^{2}(t) \leq \rho^{2}, \quad \sum_{k=1}^{M} \lambda_{k}^{r} v_{k}^{2}(t) \leq \sigma^{2}
$$

To prove the theorem it is sufficient to show that all $4 M$-dimensional vector-functions 


$$
(X(t), Y(t))=\left(x_{1}(t), x_{2}(t), \ldots x_{M}(t), y_{1}(t), y_{2}(t), \ldots y_{M}(t)\right)
$$

are not equal to zero, that is

$$
|X(t)|+|Y(t)| \neq 0,0 \leq t \leq \vartheta
$$

Denote

$$
C_{k}(t)=-R_{k} B_{k}(t) p_{k}+A_{k}(t) q_{k}, C(t)=\left(\sum_{k=1}^{M} \lambda_{k}^{r}\left|C_{k}(t)\right|^{2}\right)^{1 / 2},
$$

and define

$$
v_{k}(t)=\left\{\begin{array}{c}
\frac{\sigma C_{k}(t)}{C(t)}, 1 \leq k \leq M, C(t) \neq 0 \\
0, \quad k>M \text { or } C(t)=0 .
\end{array}\right.
$$

Note that $C(t)$ can be equal to 0 only at finite values of $t$ in $[0, T]$.

We have

$$
\begin{aligned}
\langle X(t), p\rangle+\langle Y(t), q\rangle & =\left\langle X^{0}, p\right\rangle+\left\langle Y^{0}, q\right\rangle-\int_{0}^{t} \sum_{k=1}^{M} \lambda_{k}^{r}\left\langle-R_{k} B_{k}(s) p_{k}+A_{k}(s) q_{k}, u_{k}(s)\right\rangle d s \\
& +\int_{0}^{t} \sum_{k=1}^{M} \lambda_{k}^{r}\left\langle-R_{k} B_{k}(s) p_{k}+A_{k}(s) q_{k}, v_{k}(s)\right\rangle d s,
\end{aligned}
$$

by direct computation, we get

$$
\begin{aligned}
\langle X(t), p\rangle+\langle Y(t), q\rangle= & \left\langle X^{0}, p\right\rangle+\left\langle Y^{0}, q\right\rangle-\int_{0}^{t} \sum_{k=1}^{M} \lambda_{k}^{r}\left\langle C_{k}(s), u_{k}(s)\right\rangle d s \\
& +\int_{0}^{t} \sum_{k=1}^{M} \lambda_{k}^{r}\left\langle C_{k}(s), v_{k}(s)\right\rangle d s .
\end{aligned}
$$

Using the Cauchy-Schwartz inequality and (12), we obtain

$$
\begin{aligned}
\langle X(t), p\rangle+\langle Y(t), q\rangle & =\left\langle X^{0}, p\right\rangle+\left\langle Y^{0}, q\right\rangle-\int_{0}^{t} \sum_{k=1}^{M}\left\langle\lambda_{k}^{r / 2} u_{k}(s), \lambda_{k}^{r / 2} C_{k}(s)\right\rangle d s+\sigma \int_{0}^{t} C(s) d s \\
& \geq\left\langle X^{0}, p\right\rangle+\left\langle Y^{0}, q\right\rangle-\int_{0}^{t}\left(\sum_{k=1}^{M} \lambda_{k}^{r}\left|u_{k}(s)\right|^{2}\right)^{1 / 2} C(s) d s+\sigma \int_{0}^{t} C(s) d s
\end{aligned}
$$




$$
\begin{aligned}
& \geq\left\langle X^{0}, p\right\rangle+\left\langle Y^{0}, q\right\rangle-\rho \int_{0}^{t} C(s) d s+\sigma \int_{0}^{t} C(s) d s \\
& =\left\langle X^{0}, p\right\rangle+\left\langle Y^{0}, q\right\rangle+(\sigma-\rho) \int_{0}^{t} C(s) d s .
\end{aligned}
$$

As $\left\langle X^{0}, p\right\rangle \geq 0,\left\langle Y^{0}, q\right\rangle \geq 0$ and $\rho \leq \sigma$, then we have

$$
\langle X(t), p\rangle+\langle Y(t), q\rangle \geq 0
$$

We assume the contrary. Let

$$
X(\tau)=0, \quad Y(\tau)=0
$$

at some $\tau \in[0, \vartheta]$ when the evader uses (12). Then, clearly,

$$
\langle X(\tau), p\rangle+\langle Y(\tau), q\rangle=0
$$

The following equalities

$$
\sum_{k=1}^{M}\left\langle\lambda_{k}^{r / 2} u_{k}(s), \lambda_{k}^{r / 2} C_{k}(s)\right\rangle=\left(\sum_{k=1}^{M} \lambda_{k}^{r}\left|u_{k}(s)\right|^{2}\right)^{1 / 2} C(s)=\rho C(s),
$$

in (13-a) hold if and only if

$$
u_{k}(s)=\frac{\rho C_{k}(s)}{C(s)}, \quad k=1,2, \ldots, M
$$

almost everywhere on [0, T]. From (12), (15) and (16), we get

$$
\langle X(\tau), p\rangle+\langle Y(\tau), q\rangle=\left\langle X^{0}, p\right\rangle+\left\langle Y^{0}, q\right\rangle+(\sigma-\rho) \int_{0}^{\tau} C(s) d s=0
$$

Combining (10) with (17), we find that

(i) $\rho=\sigma$, and, hence, from (16) we get

$$
u_{k}(s)=\frac{\sigma C_{k}(s)}{C(s)}, \quad k=1,2, \ldots, M,
$$

almost everywhere on $[0, \tau]$, that is, $u_{k}(s)=v_{k}(s)$ almost everywhere on $[0, \tau]$,

(ii) $\left\langle X^{0}, p\right\rangle=0,\left\langle Y^{0}, q\right\rangle=0$.

Since $u_{k}(s)=v_{k}(s), 0 \leq s \leq \tau$, then from (11), we get 


$$
\begin{gathered}
X_{k}(t)=x_{k}^{0}+\int_{0}^{t} R_{k} B_{k}(s) v_{k}(s) d s-\int_{0}^{t} R_{k} B_{k}(s) v_{k}(s) d s=x_{k}^{0}, \\
\left.Y_{k}(t)=y_{k}^{0}-\int_{0}^{t} A_{k}(s) v_{k}(s)\right) d s+\int_{0}^{t} A_{k}(s) v_{k}(s) d s=y_{k}^{0} .
\end{gathered}
$$

Consequently,

$$
X(t)=X^{0}, Y(t)=Y^{0}, 0 \leq t \leq \tau .
$$

In accordance with the choice

$$
\left|X^{0}\right|+\left|Y^{0}\right| \neq 0
$$

and therefore we have

$$
|X(t)|+|Y(t)| \neq 0,0 \leq t \leq \tau
$$

which contradicts (18).

\section{References}

1. Advonin, S.A. and Ivanov, S.A. The controllability of Systems with Distributed Parameters and Families of Exponentials. (UMKVO, Kiev, 1989).

2. Butkovskiy A.G. Control Methods in Systems with Distributed Parameters. (Nauka, Moscow, 1975).

3. Chernous'ko F.L. Bounded Controls in Systems with Distributed Parameters. Prikl. Mat. Mekh., Vol 56. No. 5. (1992), p. 810-826.

4. Pontryanin. Collected Works. (Nauka, Moscow, 1988).

5. Tukhtasinov, M. On Some Problems in the Theory of Differential Pursuit Games in Systems with Distributed Parameters. Prikl. Mat. Mekh., Vol. 59, No. 6, (1995), p. 979-984.

6. Satimov N.Yu., and Tukhtasinov M. Game problems on a fixed interval in controlled firstorder evolution equations, Mathematical notes., Vol.80, No 3-4, (2006), p.578-589.

7. Satimov N.Yu., and Tukhtasinov M. On Game Problems for Second-Order Evolution Equations. Russian Mathematics (Iz. VUZ)., Vol. 51, No.1, ( 2007), p. 49-57.

8. Ibragimov G.I. A problem of optimal pursuit in systems with distributed parameters. J. Appl. Math. Mech., Vol. 66, No 5, (2003), p. 719-724.

9. Ibragimov G.I., and Risman Mat Hasim. A Differential Game of Evasion from Many Pursuers. Malaysian Journal of Mathematical Science., Vol. 2, No.1, (2008), p. 49-58.

10. Fateh Allahabi. The Existence-Uniqueness Theorem for a System of Differential Equations in the Space $l_{r+1}^{2}$. Malaysian Journal of Mathematical Sciences., Vol. 5, No.1, (2010), p. 111123. 